Supporting Information

\title{
Comparative Analysis of Tryptophan Dynamics in Spectrin and its Constituent Domains: Insights from Fluorescence
}

\author{
Sreetama Pal ${ }^{\dagger, \pi}$, Dipayan Bose ${ }^{\ddagger, \S,}$, Abhijit Chakrabarti ${ }^{\ddagger, \S}$ and Amitabha Chattopadhyay ${ }^{\dagger, *}$ \\ ("lequal contribution)
}

${ }^{\dagger}$ CSIR-Centre for Cellular and Molecular Biology, Uppal Road, Hyderabad 500 007, India;

${ }^{\ddagger}$ Crystallography and Molecular Biology Division, Saha Institute of Nuclear Physics, 1/AF Bidhannagar, Kolkata 700 064, India; ${ }^{\S}$ Homi Bhabha National Institute, Anushaktinagar, Mumbai 400 094, India

*Address correspondence to Amitabha Chattopadhyay;

E-mail: amit@ ccmb.res.in, Phone: +91-40-2719-2578 
S1. Steady state fluorescence measurements. Steady state fluorescence measurements were acquired with a Fluorolog-3 Model FL3-22 spectrofluorometer (Jobin Yvon, Edison, NJ) at room temperature $\left(\sim 23^{\circ} \mathrm{C}\right)$ using semi-micro quartz cuvettes. Slit widths of 2 and $4 \mathrm{~nm}$ were used for excitation and emission, respectively. Spectra were recorded in the corrected spectrum mode. Contributions from solvent Raman peaks and other scattering artifacts were corrected by subtraction of background intensities (from samples without peptide) from sample spectra. Data shown is representative of at least three independent measurements. Fluorescence emission maxima were assigned based on the centre of mass of each spectrum and in each case, the observed emission maximum was identical to or within $\pm 1 \mathrm{~nm}$ of the reported values.

Values of fluorescence anisotropy were calculated using the following equation: ${ }^{1}$

$$
r=\frac{\mathrm{I}_{\mathrm{VV}}-\mathrm{GI}_{\mathrm{VH}}}{\mathrm{I}_{\mathrm{VV}}+2 \mathrm{GI}_{\mathrm{VH}}}
$$

where $\mathrm{IVV}_{\mathrm{VV}}$ and $\mathrm{I}_{\mathrm{VH}}$ represent fluorescence intensities (after appropriate background subtraction) measured with a configuration consisting of vertically oriented excitation polarizer and vertically and horizontally oriented emission polarizer, respectively. G denotes the grating correction factor and is the ratio of efficiencies of the system for the detection of vertically and horizontally polarized light $\left(\mathrm{I}_{\mathrm{HV}} / \mathrm{I}_{\mathrm{HH}}\right)$. Data shown is representative of at least three independent measurements.

S2. Time-resolved fluorescence measurements. Time-resolved fluorescence intensity decays were acquired using a Delta-D TCSPC system (Horiba Jobin Yvon IBH, Glasgow, UK) with EzTime software version 3.2.2.4 (Horiba Scientific, Edison, NJ) in the time-correlated single photon counting (TCSPC) mode, as described earlier. ${ }^{2}$ In order to optimize the signal-to-noise ratio, 10,000 photon counts were collected at the peak channel. Data acquisition was carried out with an emission band pass of $4 \mathrm{~nm}$ or less and a neutral density filter (ND1) in the excitation path. The sample and scatterer profiles were acquired in 10 alternate cycles of 1,000 photon counts (10\% 
acquisition) at each of the peak channels, to compensate for shape and timing drifts that could occur during data acquisition. This arrangement has the additional advantage of preventing prolonged exposure and subsequent photodamage of the fluorophore.

Data were stored and analyzed using in-built plugins in the EzTime software version 3.2.2.4 (Horiba Scientific, Edison, NJ). Fluorescence intensity decay curves were deconvoluted with the IRF and analyzed as a sum of exponential terms given by the equation:

$$
\mathrm{F}(\mathrm{t})=\Sigma_{\mathrm{i}} \alpha_{\mathrm{i}} \exp \left(-\mathrm{t} / \tau_{\mathrm{i}}\right)
$$

where $F(t)$ is the fluorescence intensity at time $t$ and $\alpha_{i}$ is a pre-exponential factor denoting the fractional contribution to the time-resolved decay of the $i^{\text {th }}$ component with a lifetime of $\tau_{i}$, such that $\Sigma_{i} \alpha_{i}=1$. The decay parameters were recovered using a nonlinear least squares iterative fitting program based on the Marquadt algorithm. ${ }^{3}$ The program also includes subroutine packages associated with statistical analysis and plotting. ${ }^{4}$ The goodness of fit of a given data set to a chosen function was evaluated by the $\chi^{2}$ values, the weighted residuals ${ }^{5}$ and the autocorrelation function of the weighted residuals. ${ }^{6}$ A fit was considered acceptable when plots of the weighted residuals and their autocorrelation function exhibited random deviation about zero, with a $\chi^{2}$ value of not more than 1.2. Intensity-averaged mean fluorescence lifetimes $(<\tau>)$ for triexponential fluorescence decays were calculated from the decay times and pre-exponential factor using the equation: ${ }^{1}$

$$
\langle\tau\rangle=\frac{\alpha_{1} \tau_{1}^{2}+\alpha_{2} \tau_{2}^{2}+\alpha_{3} \tau_{3}^{2}}{\alpha_{1} \tau_{1}+\alpha_{2} \tau_{2}+\alpha_{3} \tau_{3}}
$$

Fluorescence lifetimes were represented as intensity-averaged mean values since this parameter does not depend on the method of analysis employed and the number of exponential terms used to deconvolute the time-resolved fluorescence decay. 
S3. Estimation of apparent rotational correlation time. Apparent rotational correlation times were calculated using Perrin's equation: ${ }^{1}$

$$
\tau_{\mathrm{c}}=\frac{\mathrm{r}<\tau>}{\mathrm{r}_{\mathrm{o}}-\mathrm{r}}
$$

where $r_{o}$ is the fundamental anisotropy of tryptophan residues, $r$ corresponds to fluorescence anisotropy (from Figure 2a), and $\langle\tau>$ is the intensity-averaged mean fluorescence lifetime (from Figure $2 \mathrm{~b}$ and Table 1). Fluorescence anisotropy and lifetime data were collected at the respective excitation wavelengths of 295 and $297 \mathrm{~nm}$, while the emission wavelength was set to the emission maxima of each protein (shown in insets to Figure $1 \mathrm{~b}$ and $1 \mathrm{c}$ for native and denatured conditions, respectively). The value of $r_{o}$ was taken to be 0.16 for tryptophan residues. ${ }^{7}$ The relative change in apparent rotational correlation time was calculated using the following equation:

$$
\Delta \tau_{\mathrm{c}}(\%)=\left(\left(\tau_{\mathrm{c}, \text { native }}-\tau_{\mathrm{c}, \text { denatured }}\right) / \tau_{\mathrm{c}, \text { native }}\right) \times 100
$$

where $\tau_{\mathrm{c} \text {,native }}$ and $\tau_{\mathrm{c} \text {,denatured }}$ are the apparent rotational correlation time characteristic of tryptophan residues in spectrin and its domains in native (filled bars in Figure 4) and denatured (hatched bars in Figure 4) conditions, respectively. 
Figure S1

Pal et al.

(a)

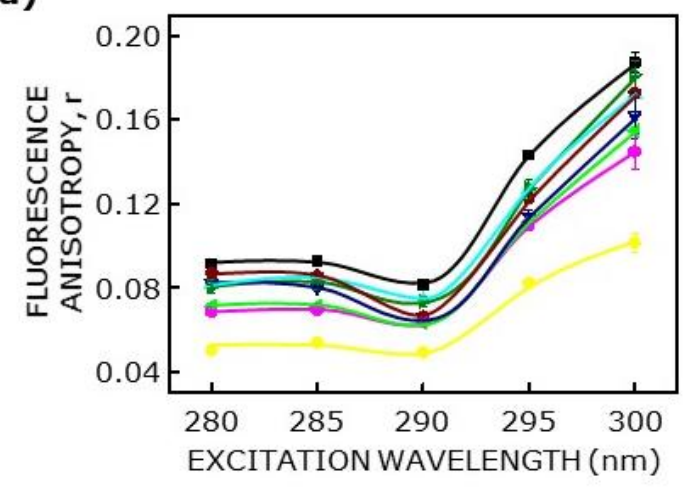

(c)

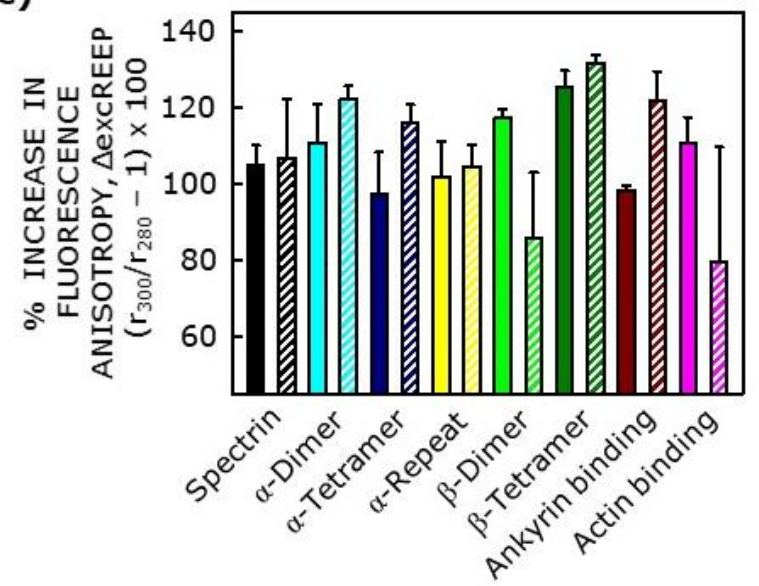

(b)

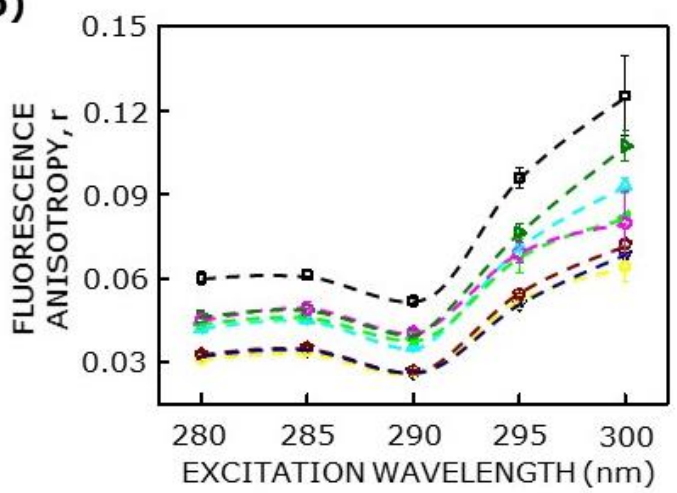

(d)

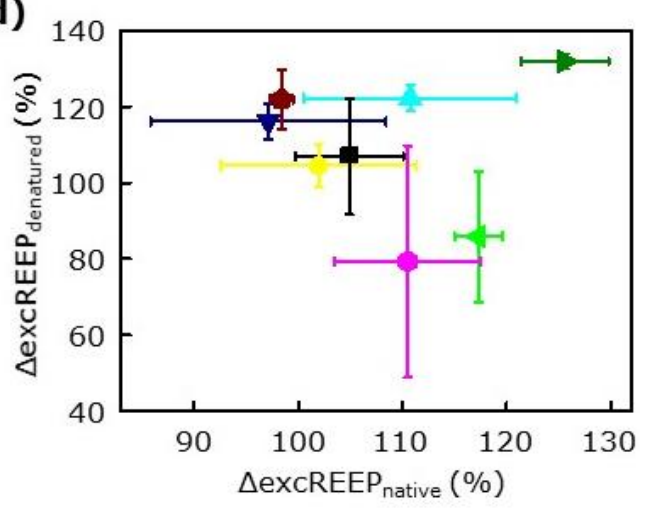

Figure S1. Effect of changing excitation wavelength on fluorescence anisotropy of tryptophan residues in spectrin and its constituent domains in (a) native (closed symbols) and (b) denatured (open symbols) conditions. The emission wavelength was set to the emission maxima of each protein (shown in insets to Figure $1 \mathrm{~b}$ and $1 \mathrm{c}$ for native and denatured conditions, respectively). Fluorescence anisotropy values were calculated using eq S1. Lines joining the data points are provided merely as viewing guides. (c) Corresponding changes in $\triangle$ excREEP, defined as the percentage increase in anisotropy at $300 \mathrm{~nm}$ relative to that at $280 \mathrm{~nm}$, for tryptophan residues in spectrin and its domains in native (filled bars) and denatured (hatched bars) conditions. The concentration of spectrin and each spectrin domain was 0.25 and $20 \mu \mathrm{M}$, respectively. Color coding and all other experimental conditions are the same as in Figure 1b and 1c. (d) Scatter plot of $\triangle$ excREEP for tryptophan residues of spectrin and its domains in native $v s$. denatured conditions. Data represent means $\pm \mathrm{SE}$ of at least three independent measurements. See Section S1 for more details. 
Figure S2

Pal et al.

(a)

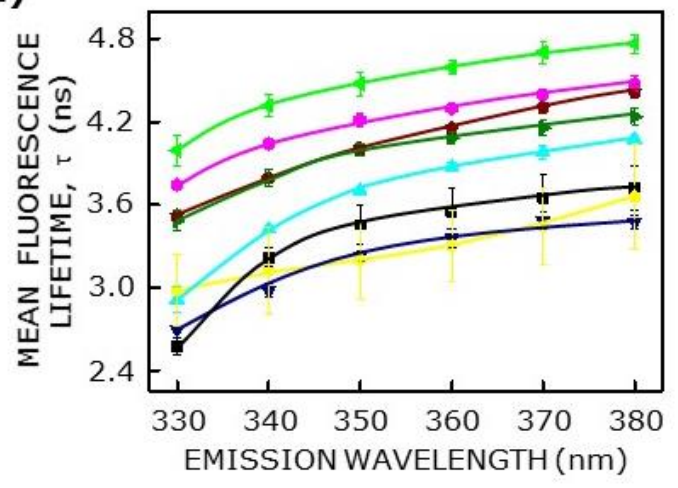

(c)

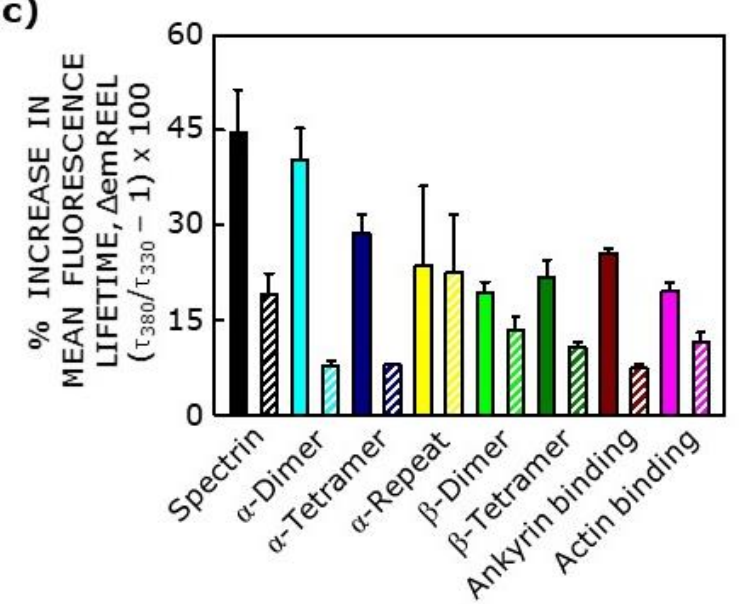

(b)

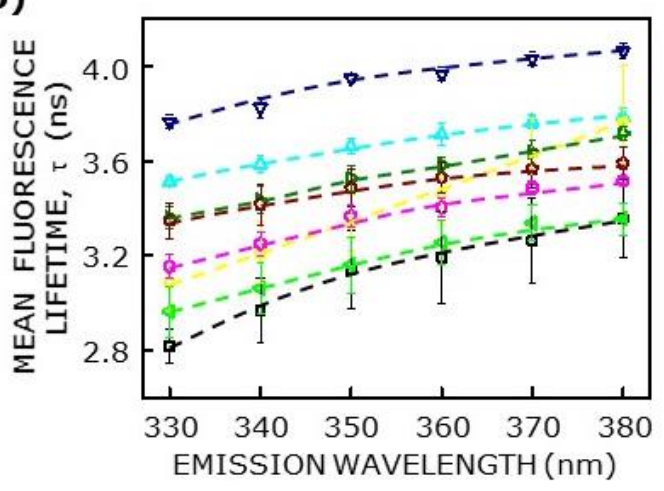

(d)

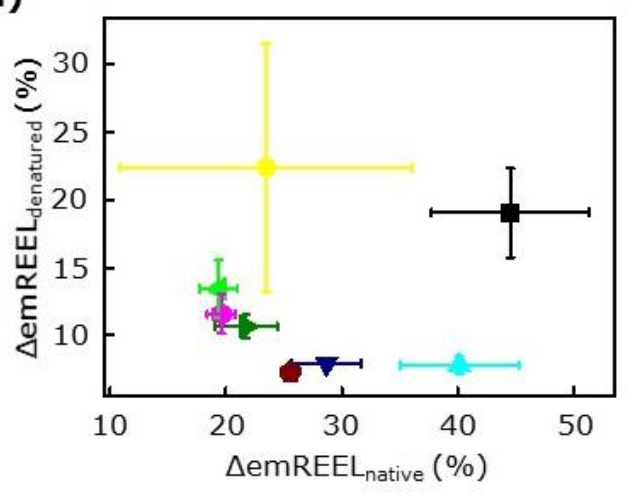

Figure S2. Effect of changing emission wavelength on mean fluorescence lifetime of tryptophan residues in spectrin and its constituent domains in (a) native (closed symbols) and (b) denatured (open symbols) conditions. The excitation wavelength was $297 \mathrm{~nm}$ in all cases. Intensityaveraged mean fluorescence lifetimes were calculated using eq S3. Lines joining the data points are provided merely as viewing guides. (c) Corresponding changes in $\triangle$ emREEL, defined as the percentage increase in intensity-averaged mean fluorescence lifetime at $380 \mathrm{~nm}$ relative to that at $330 \mathrm{~nm}$, for tryptophan residues in spectrin and its domains in native (filled bars) and denatured (hatched bars) conditions. The concentration of spectrin and each spectrin domain was 0.25 and $20 \mu \mathrm{M}$, respectively. Color coding and all other experimental conditions are the same as in Figure $1 b$ and 1c. (d) Scatter plot of $\triangle$ emREEL for tryptophan residues of spectrin and its domains in native vs. denatured conditions. Data represent means \pm SE of at least three independent measurements. See Section S2 for more details 
Figure S3

Pal et al.

(a)

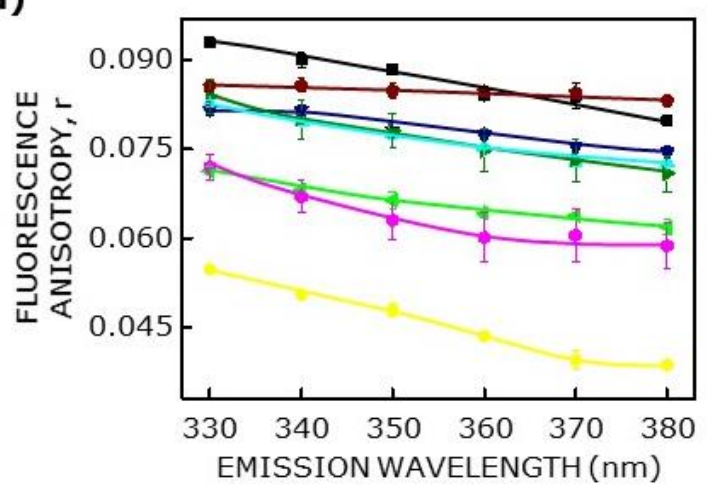

(c)

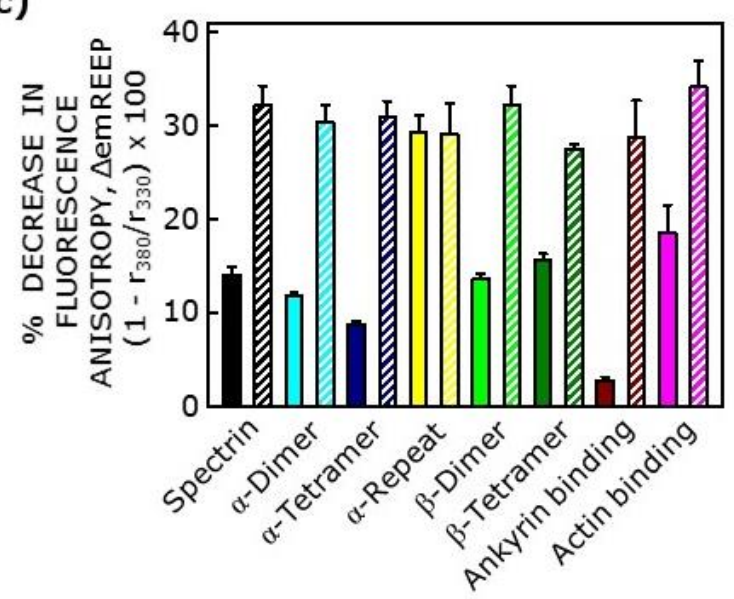

(b)

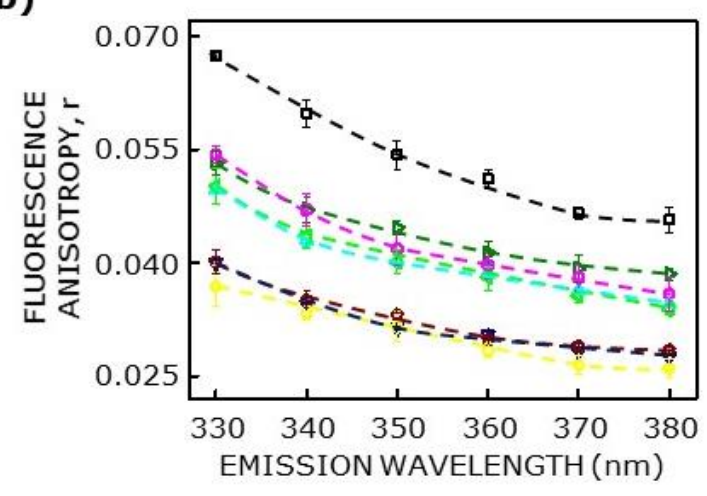

(d)

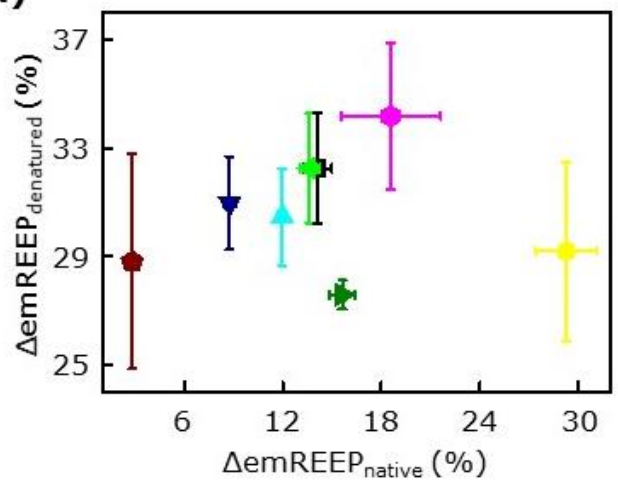

Figure S3. Effect of changing emission wavelength on fluorescence anisotropy of tryptophan residues in spectrin and its constituent domains in (a) native (closed symbols) and (b) denatured (open symbols) conditions. Excitation wavelength was kept constant at $280 \mathrm{~nm}$. Fluorescence anisotropy values were calculated using eq S1. Lines joining the data points are provided merely as viewing guides. (c) Corresponding changes in $\triangle$ emREEP, defined as the percentage decrease in fluorescence anisotropy at $380 \mathrm{~nm}$ relative to that at $330 \mathrm{~nm}$, for tryptophan residues in spectrin and its domains in native (filled bars) and denatured (hatched bars) conditions. The concentration of spectrin and each spectrin domain was 0.25 and $20 \mu \mathrm{M}$, respectively. Color coding and all other experimental conditions are the same as in Figure $1 \mathrm{~b}$ and 1c. (d) Scatter plot of $\triangle$ emREEP for tryptophan residues of spectrin and its domains in native $v s$. denatured conditions. Data represent means \pm SE of at least three independent measurements. See Section S1 for more details. 


\section{REFERENCES}

1. Lakowicz, J. R. Principles of Fluorescence Spectroscopy; Plenum Press: New York, 1983.

2. Pal, S.; Aute, R.; Sarkar, P.; Bose, S.; Deshmukh, M. V.; Chattopadhyay, A. Constrained dynamics of the sole tryptophan in the third intracellular loop of the serotonin ${ }_{1 \mathrm{~A}}$ receptor. Biophys. Chem. 2018, 240, 34-41.

3. Bevington, P. R. Data Reduction and Error Analysis for the Physical Sciences; McGrawHill: New York, 1969.

4. O'Connor, D. V.; Phillips, D. Time-correlated Single Photon Counting; Academic Press: London, 1984, 180-189.

5. Lampert, R. A.; Chewter, L. A.; Phillips, D.; O'Connor, D. V.; Roberts, A. J.; Meech, S. R. Standards for nanosecond fluorescence decay time measurements. Anal. Chem. 1983, 55, 68-73.

6. Grinvald, A.; Steinberg, I. Z. On the analysis of fluorescence decay kinetics by the methos of least-squares. Anal. Biochem. 1974, 59, 583-598.

7. Eftink, M. R.; Selvidge, L. A.; Callis, P. R.; Rehms, A. A. Photophysics of indole derivatives: experimental resolution of $\mathrm{L}_{a}$ and $\mathrm{L}_{b}$ transitions and comparison with theory. $J$. Phys. Chem. 1990, 94, 3469-3479. 